\title{
Generalized Sharing in Survivable Optical Networks
}

\author{
Maher Ali, Senior Member, IEEE
}

\begin{abstract}
Shared path protection has been demonstrated to be a very efficient survivability scheme for optical networking. In this scheme, multiple backup paths can share a given optical channel if their corresponding primary routes are not expected to fail simultaneously. The focus in this area has been the optimization of the total channels (i.e., bandwidth) provisioned in the network through the intelligent routing of primary and backup routes. In this work, we extend the current path protection sharing scheme and introduce the Generalized Sharing Concept. In this concept, we allow for additional sharing of important node devices. These node devices (e.g., optical-electronic-optical regenerators (OEOs), pure all-optical converters, etc.) constitute the dominant cost factor in an optical backbone network and the reduction of their number is of paramount importance. For demonstration purposes, we extend the concept of $1: N$ shared path protection to allow for the sharing of electronic regenerators needed for coping with optical transmission impairments. Both design and control plane issues are discussed through numerical examples. Considerable cost reductions in electronic budget are demonstrated.
\end{abstract}

Index Terms-Optical networks, shared protection.

\section{Generalized Sharing OF Network Resources}

$\mathbf{S}$ HARED path protection has been established as a mechanism for providing considerable savings in terms of the number of provisioned wavelengths [1]-[6]. In this protection scheme, optical services whose primary routes are physically diverse, can be allowed to share protection wavelengths (on their protection paths) on some of the fiber links. This can be accomplished since, under the single-link failure scenario, physically diverse primary routes will not fail at the same time; hence their protection paths will not be activated simultaneously. Previous work on shared protection considered the wavelength (or bandwidth) as the only shared resource that needs to be optimized. This focus ignores the fact that the dominant cost of optical backbone networks is that of the optical-electronic-optical (OEO) devices [1] that are needed for dealing with optical transmission impairments and/or realization of wavelength conversion [10]. In this work, we introduce the Generalized Sharing Concept which allows for the sharing of not only fiber resources, but also valuable node equipment. In this context, lightpaths [11] can share wavelengths and regeneration points on their protection paths. Motivated by this new sharing concept, we study the optimization of provisioned OEOs in a survivable optical network. In such a network, a lightpath is routed all-optically as far as possible and it encounters regeneration at intermediate nodes when the transmission impairments significantly degrade

Manuscript received December 2, 2003; revised June 8, 2005; approved by IEEE/ACM TRANSACTIONS ON NETWORKING Editor C. Qiao.

The author is with Alcatel Research and Innovation, Plano, TX 75075 USA (e-mail: maher.ali@alcatel.com).

Digital Object Identifier 10.1109/TNET.2006.886293

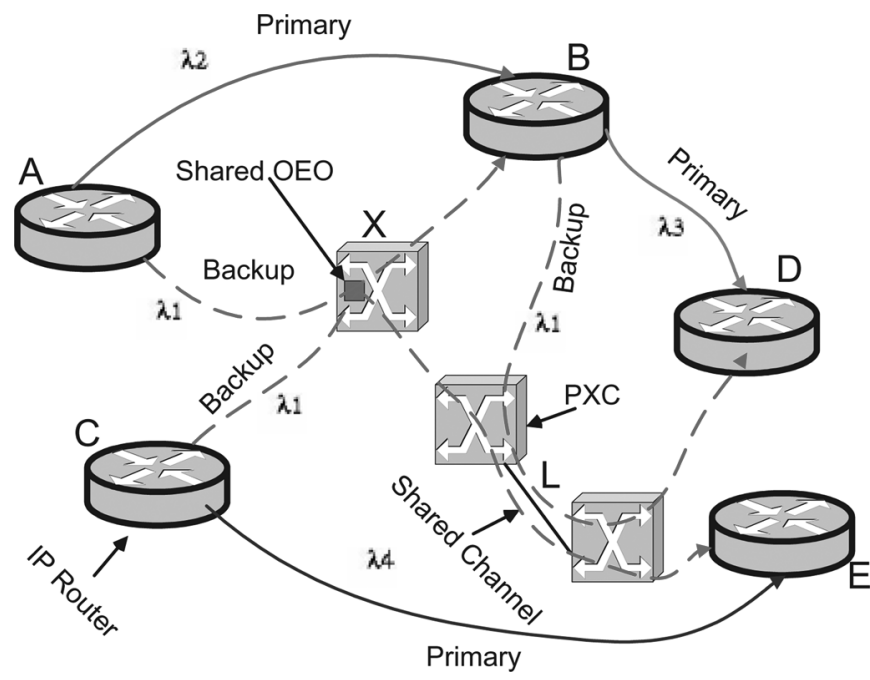

Fig. 1. Example illustrating the generalized sharing concept. IP routers are interconnected via switching nodes. Each switching node is capable of all-optical switching using a photonic cross-connect (PXC) and optional OEOs used in regeneration/wavelength conversion. The example shows two backup lightpaths sharing an OEO at Node $X$. In addition, $\lambda_{1}$ on Fiber $L$ is shared by two backup lightpaths.

the signal [12], [13]. After that, we address some of the implications on the control plane.

\section{A. Illustration of the Concept}

Fig. 1 illustrates the idea behind the generalized sharing concept. Three protected lightpaths are established: $(A, B),(C, E)$, and $(B, D)$. Since the primary routes do not have physical links in common, the resource(s) on their respective backup paths can be shared. In the figure, we notice that the backup paths for connections $(C, E)$ and $(A, B)$ share an OEO at Node $X$. When the primary of either lightpaths fails, their corresponding backup path is activated and it can use the regeneration node. Without this sharing, the two backup paths, which each require an OEO at Node $X$ to clean up their signal from accumulated optical impairments, may potentially need to have a dedicated OEO. The other shared resource is the wavelength and is shown in the figure where Channel $\lambda_{1}$ on Link $L$ is shared between connections $(C, E)$ and $(B, D)$. In general, $n$ backup routes can share the same resource if their primary routes are physically diverse.

For every shared object in the network, a sharing table must be employed. This sharing table contains an identification of the object being shared as well as a list of fibers' identification numbers. This list is formed by the union of the fibers traversed by the primary paths of all services whose backup routes share that object. Every backup path that wants to share an object must have the property that its primary route does not traverse any of the fibers of that object's sharing table. We utilize two sharing 


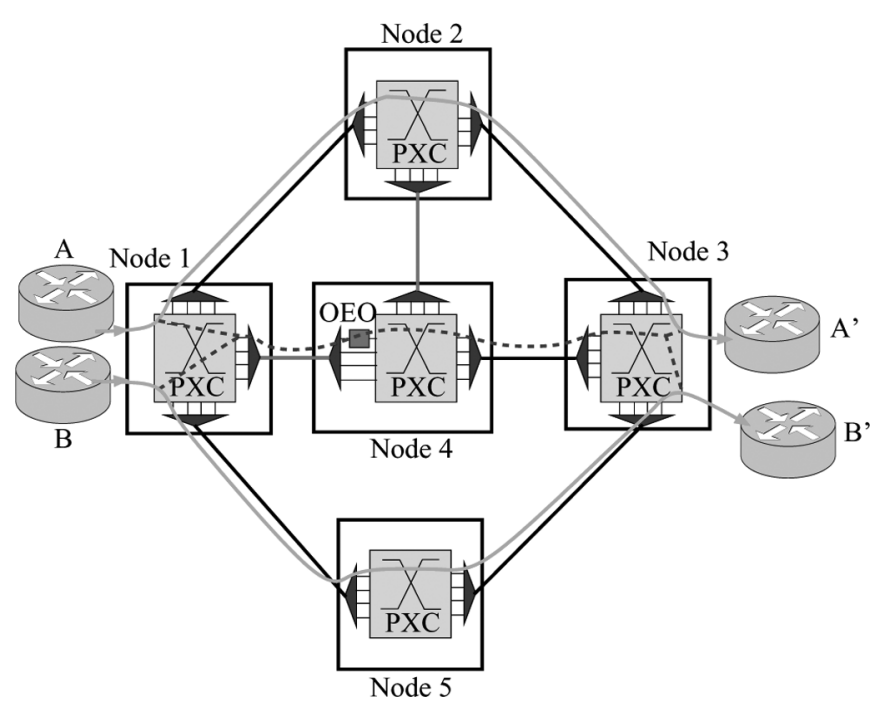

Fig. 2. Illustration of Level 1 sharing. The two backup paths share a fiber terminated by an OEO regenerator at Node 4. The network example shows IP routers interconnected via a mesh of switching nodes. Each switching node is comprised of a photonic cross-connect (PXC), OEOs, and multiplexers.

tables types: a sharing table of the channels and a sharing table of the OEOs.

It is worth noting that no additional device is needed for the realization of OEO sharing, since as it will be shown, the optical switching fabric directs the specific backup signal to a pool of shared OEOs when failure occurs.

In the following, we provide details on how the sharing scheme extended to OEOs can be realized.

\section{B. Two Levels For Sharing a Regenerator}

In practice, one can think of two sharing levels (schemes) that govern the sharing of OEO devices at intermediate switching nodes. The first scheme (Level 1 sharing) is an extension of the currently used wavelength-sharing scheme used in bandwidth optimization. In this scheme, a channel, say $\lambda_{i}$ on Fiber $(x, y)$, requires the reservation of an OEO device at Node $y$ if one or more of the protection paths sharing Channel $\lambda_{i}$ requires OEO regeneration. This requirement for OEO regeneration is dictated by the need for signal recovery, due to accumulated optical impairments, and/or the need for wavelength conversion, due to the unavailability of Channel $\lambda_{i}$ on the next hop. In either case, once it is decided that one of the protection services needs an OEO at a node, all new protection services that share that channel can use this OEO device with zero additional cost. Fig. 2 shows an illustration of Level 1 sharing. In the example, two fiber-disjoint primary paths are established: 1) \{Client A, Node 1, Node 2, Node 3, Client $\left.A^{\prime}\right\}$, and 2) $\left\{\right.$ Client $B$, Node 1, Node 5 , Node 3 , Client $\left.B^{\prime}\right\}$. Under single link-failure scenario, one observes that since the lightpaths are fiber-disjoint, both primary lightpaths cannot fail at the same time; hence at most one of their protection lightpaths will be activated. In the figure, both protection paths are routed on $\{$ Node 1, Node 4, Node 3$\}$. Let us assume that the transmission quality of the transmission system between Nodes

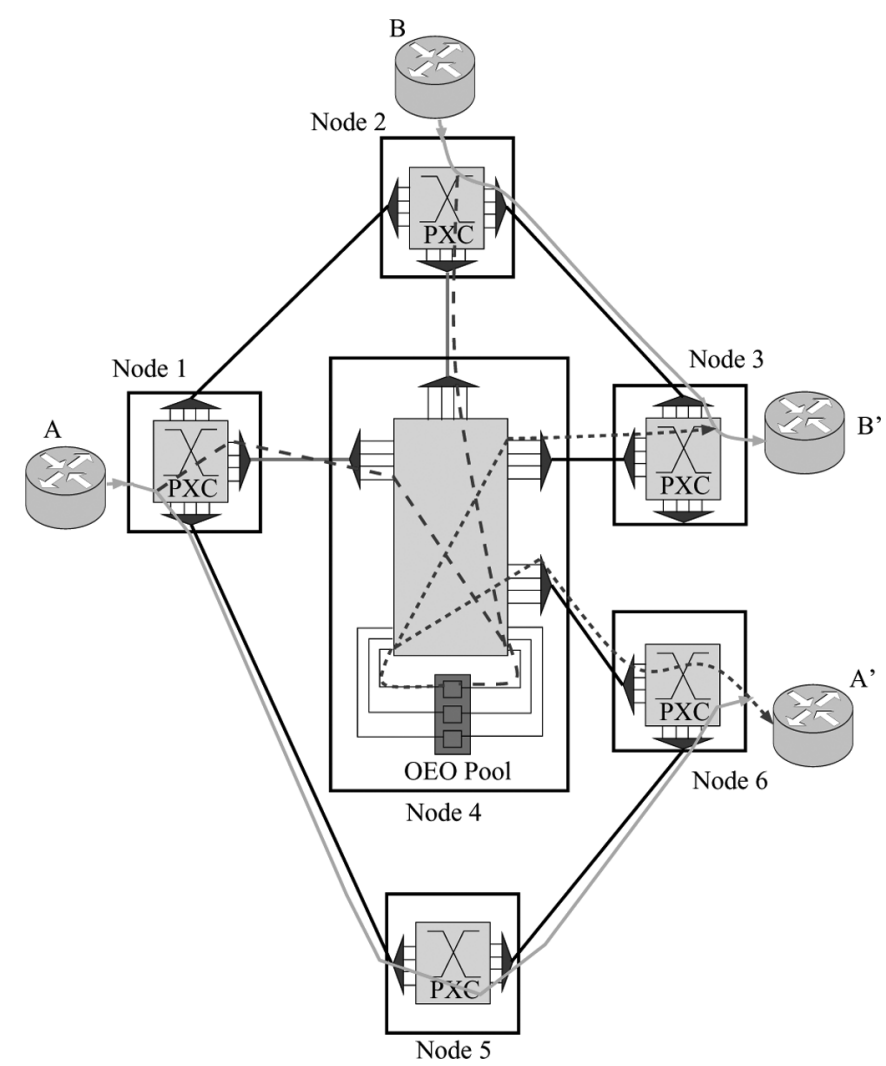

Fig. 3. Illustration of Level 2 sharing. The two backup paths share a regenerator at intermediate Node 4.

1 and 4 is poor that it requires signal regeneration at Node 4 . In this case, one OEO device can be reserved to accommodate up to $n$ (in this case $n=2$ ) protection paths.

The second scheme for sharing OEO devices is Level 2 sharing. In Level 2, two protection paths whose primary paths are fiber-disjoint can share an OEO device at a given intermediate node irrespective of the bandwidth (channel) sharing. Fig. 3 illustrates the idea. Two primary lightpaths $\left\{\right.$ Client $A$, Node 1, Node 5, Node 6, Client $\left.A^{\prime}\right\}$ and $\left\{\right.$ Client $B$, Node 2, Node 3, Client $\left.B^{\prime}\right\}$ are fiber-disjoint. Their respective protection paths are $\left\{\right.$ Client A, Node 1, Node 4, Node 6, Client $\left.A^{\prime}\right\}$ and $\left\{\right.$ Client B, Node 2, Node 4, Node 3, Client $\left.B^{\prime}\right\}$ share an OEO device at Node 4 even though the two input signals at Node 4 come from two distinct input fibers and exit at two different fibers. At the time of the activation of either protection signal, the signal is diverted to an OEO bank where it receives regeneration and then it is switched back in the optical domain to its output fiber. Thus, in this architecture, routes which do not share any fiber on their backups can still share OEOs on intermediate nodes. This allows for maximum sharing optimization.

Clearly, if the input nodal degree of every node in the network is equal to 1 , Level 2 degenerates to Level 1 . However, real life networks have input nodal degree that is greater or equal to 2 allowing for the utilization of this intra-fiber OEO sharing as it will be demonstrated in numerical examples. 


\section{Basic Optimization Problem}

In the following, we give a definition of the basic optimization problem discussed in this work. This problem will be used for both the design and protocol issues discussed in the following sections.

Definition Pair of Disjoint Paths with Minimum Total OEOs (PDP-OEO) Problem

The PDP-OEO problem is defined as follows. Given: a) a set of nodes and a set of fiber links connecting these nodes, b) wavelength availability on fiber links and sharing tables on every node, and c) a unidirectional connection request from $s$ to $d$. The objective is to find two fiber-disjoint paths between $s$ and $d$ such that the total number of regenerators needed to cope with transmission impairments is minimized.

It is worth noting that in this work we focus primarily on transmission impairments as the only need for OEOs. However, the methodology developed in this work can be equally applied to wavelength converters needed for coping with the wavelength continuity constraint. This later problem is left for future research. In the following section, we provide a solution for the above problem and use that solution for the embedding of a virtual topology (i.e., set of demands) on a given physical topology with minimum number of OEOs. This solution is also used for the dynamic provisioning of lightpath services.

\section{Network DESIGN PERFORMANCE StUdy}

In this section, we focus on the design problem motivated by the generalized sharing concept. Informally, given the network physical topology and a set of network connections representing the virtual topology required, the objective is establish all connections with their protection paths such that the total number of OEOs in the network is minimized. In the following, we discuss some design algorithms that can be used to optimize the network cost. Next, we provide a simple example illustrating this issue. After that, a more realistic network is considered.

\section{A. Network Design Issues}

The network design problem is given by the following:

Definition: Survivable Network Design With Minimum OEOs (SND-OEO).

Given: 1) a network physical topology represented by a graph $G=(V, L)$ composed of the set of nodes $V$ and the set of fibers $L$ connecting these nodes with the set of available wavelengths on a fiber given by $W$, and 2) a set of demand requests $Q$, where $\psi_{i}=\left(s_{i}, d_{i}\right) \in Q$ is a unidirectional demand request for a lightpath from $s_{i}$ to $d_{i}$. The objective is to minimize the total number of OEOs used in routing both primary and backup paths for the demand in $Q$. The pseudo-code in Fig. 4 provides a heuristic for this problem.

The heuristic optimizes the OEO resources in two phases. In phase I (H-Basic), a sequential establishment of sessions is performed. As sessions are established, more opportunities for sharing OEOs become available. The main component of this phase is the EstablishSession function shown in Fig. 5.

EstablishSession() employs Best-Fit (BF) heuristic for the establishment of the primary and backup lightpaths. It goes

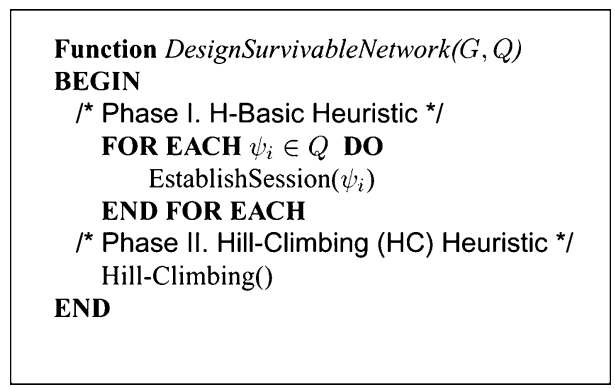

Fig. 4. Network design algorithm.

through all $|W| \times|W|$ different wavelengths for the primary and backup paths and finds the two routes for primary and backup using Wavelengths $\lambda_{p}$ and $\lambda_{b}$, respectively. In testing each $\lambda_{p}$ and $\lambda_{b}$ pair, we attempt first at finding the primary path and then finding the backup path. The pseudo-code in Fig. 6 finds the cost for establishing the primary path.

For finding the primary path, the algorithm constructs a graph $G_{1}$ such that two nodes are connected iff Wavelength $w$ is available. It labels each edge of the graph such that fibers with higher impairments and/or more shared channels have less chance of being picked up by the routing algorithm. We achieve this by labeling an Edge $e$ with $\operatorname{Imp}(e) \times(1+$ SharedWaves $(e))$, where $\operatorname{Imp}(e)$ is the amount of impairments on Fiber $e$ and SharedWaves $(e)$ is the number of wavelengths used for backup on $e$. By this formula, we hope that the shortest path algorithm avoids using shared resources (e.g., wavelengths) that may be helpful in minimizing the cost for the backup path. Dijkstra's shortest path algorithm is run on this new graph to find the shortest path from $s_{i}$ to $d_{i}$.

Let SharingTable $(e, w)$ be the sharing table for the wavelength $w$ on link $e$. In other words, it holds the set of all links in all primary routes whose backup routes use (i.e., share) wavelength $w$ on link e. Let OEOSharingTable $(i, w)$ be the sharing table for the OEO of wavelength $w$ at node $i$. Similar to the case of the wavelength sharing table, it contains all links in all primary routes whose backup routes use (i.e., share) the OEO of wavelength $w$ at node $i$. After finding the primary path, we aim at finding the backup path (Fig. 7). This backup path can only use edges that are not used by the primary path. In addition, if Wavelength $w$ is being used for backup and the primary path shares one or more fibers with the sharing table of Wavelength $w$ on $e$, the edge is excluded from the computation. Each edge $e=(i, j)$ is labeled with 0 if an OEO is available for free use at Node $j$. Otherwise, $e$ is labeled by $\operatorname{Imp}(e)$. The labeling is used to favor fibers ending with a free regenerator and at the same time minimize the impairments. Dijkstra's shortest path algorithm is run on this new graph to find the shortest path from $s_{i}$ to $d_{i}$. If such a path exists, we determine the placement of regeneration points at intermediate nodes as follows. Using RegensOnBackup() and starting with the ingress node and moving downstream on the path towards egress node, the amount of accumulated impairments is recorded as well as the location of the last regenerator that can be shared (here differences between Levels 1 and 2 determine whether an OEO can be shared or not). 


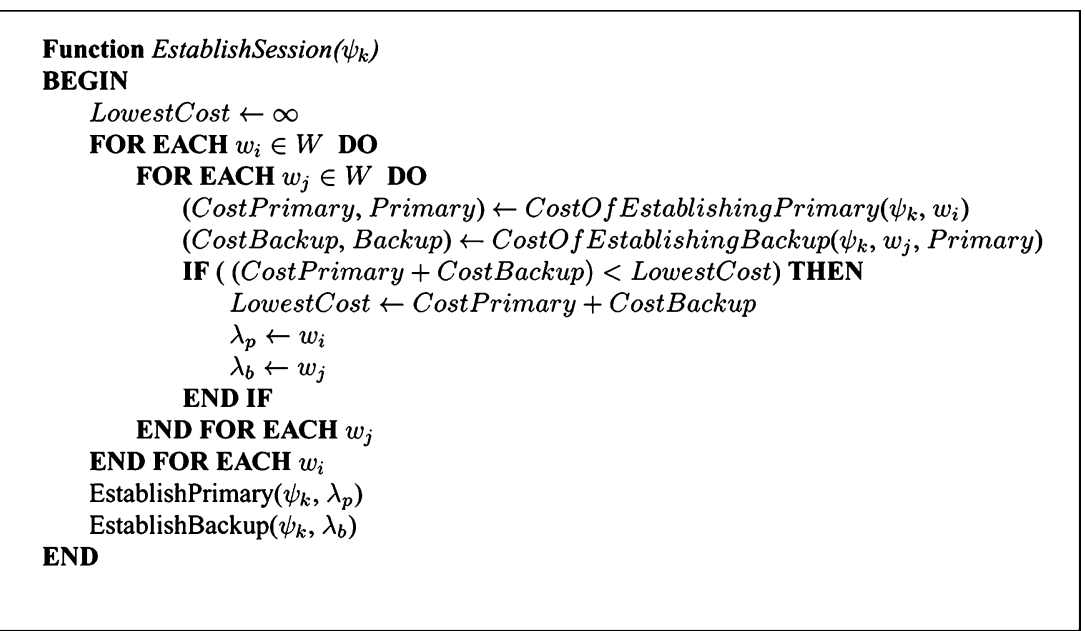

Fig. 5. Establish a session heuristic.

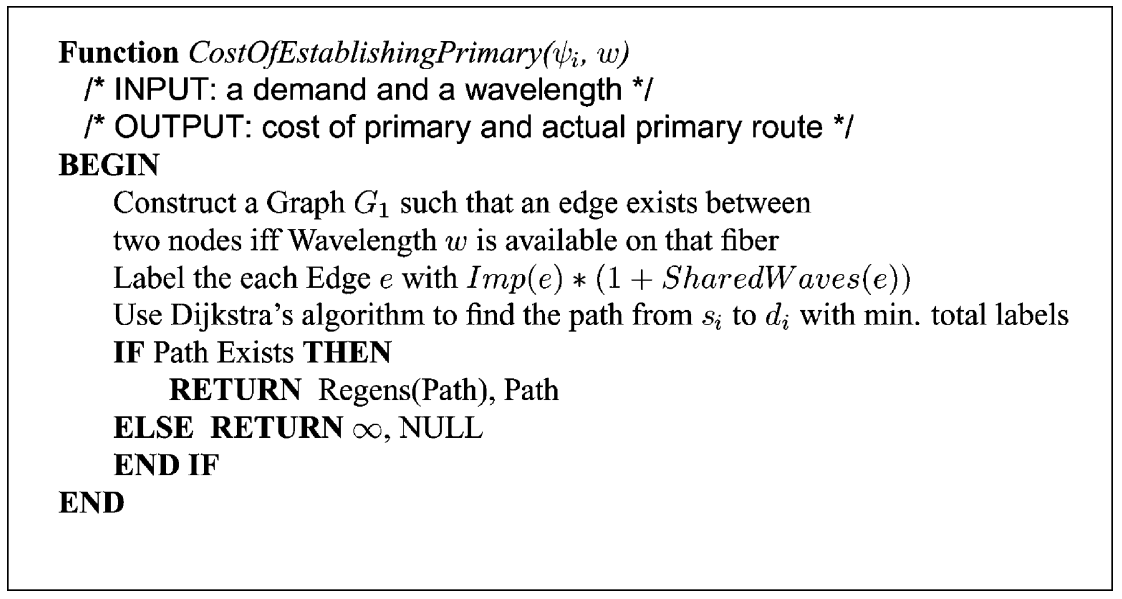

Fig. 6. Cost of establishing primary path for a session.

Whenever the impairments reach the threshold, the last shared regenerator is used to clean up the signal. If no such regenerator can be shared, a new regenerator is installed. The process is repeated until we reach the egress node. Total cost is composed of only newly added regenerators.

It is worth noting that this sequential approach (i.e., fixing a primary route and then looking for the backup) may sometime result in a trap situation, in which a pair of diverse pair of primary and backup paths cannot be found, even if one exists, because the primary was not correctly selected. Finding an optimum solution is unfortunately an intractable problem, due to the fact that different cost-metrics are used for computing the primary and the backup. For more information on this problem and possible remedies, please refer to [14] and [15].

The time complexity for establishing one session is $O\left(|W|^{2} \times\right.$ $\left.\left(|V|^{2}+|L|\right)\right)$ assuming the shortest path is computed in $O\left(|V|^{2}\right)$ and labeling an auxiliary graph and installing regenerators is done in $O(|L|)$. For larger values of $|W|$, one can replace the Best-Fit approach with a scheme that is not as computationally-intensive (such as First-Fit) to achieve time complexity of $O\left(|W| \times\left(|V|^{2}+|L|\right)\right)$.

After the sequential establishment of the sessions, we use the second phase to further optimize the resources. In Phase II, the well-known Hill-Climbing (HC) algorithm is used to optimize the solution in Phase I. At each step of the HC heuristic, all sessions are inspected at a time. For each session, we find its current cost composed of OEOs on primary and backup routes. The session is then removed and resources are freed. Next, the session is established by taking advantage of all sharing resources available. If the new cost (which is computed as in Phase I) is less than the previous cost, a profit is made. If the current profit is larger than the profit achieved so far, the session becomes the candidate for re-routing. At the end of inspecting all sessions, the candidate session whose re-routing provides the maximum profit is re-routed. This step is repeated until there is no candidate session for re-routing (i.e., no further enhancements to the number of OEOs provisioned). Fig. 8 shows a pseudo-code for the HC heuristic.

\section{B. A Simple Example}

In the following, we present an example showing both the benefits of shared protection compared with $1+1$ dedicated path protection and the cost reduction using Level 2 sharing. Consider a simple physical topology of an 8-node network as shown in Fig. 9. The network consists of optical cross-connects (photonic switches with regeneration capability) connected via 13 


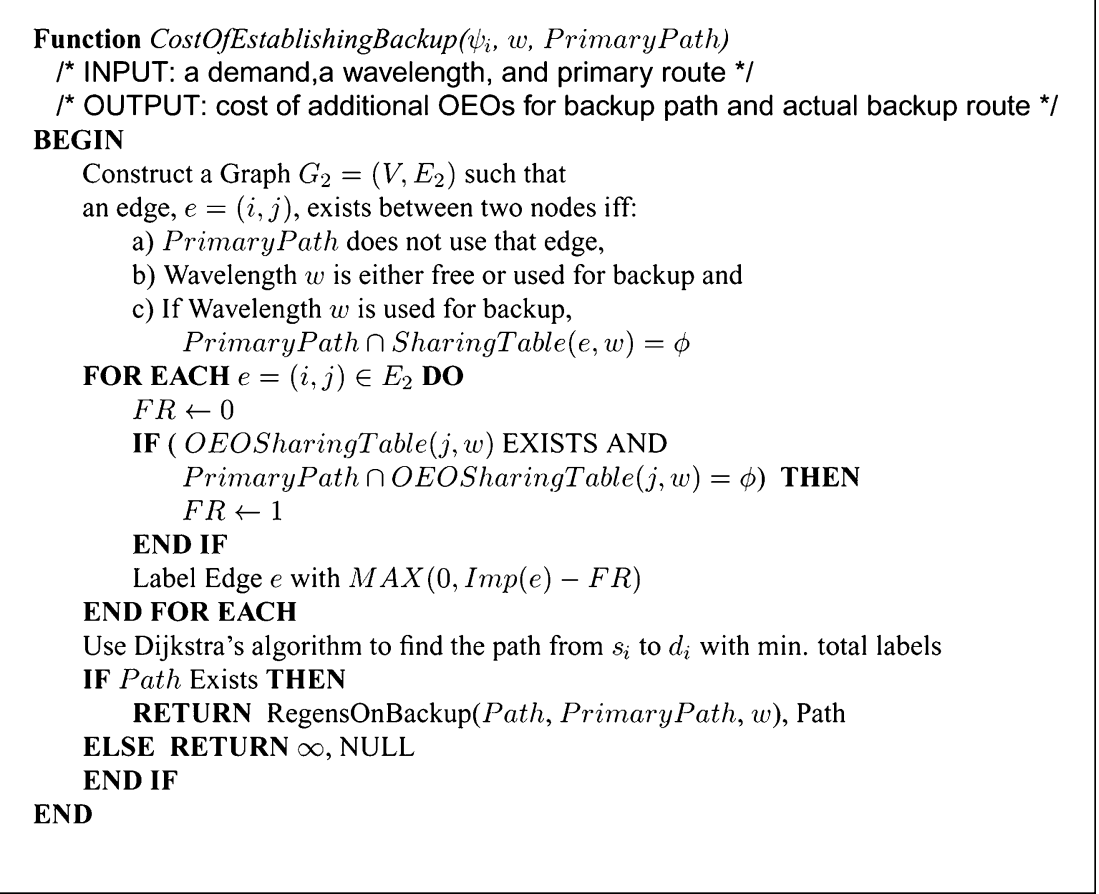

Fig. 7. Cost of establishing backup path for a session.

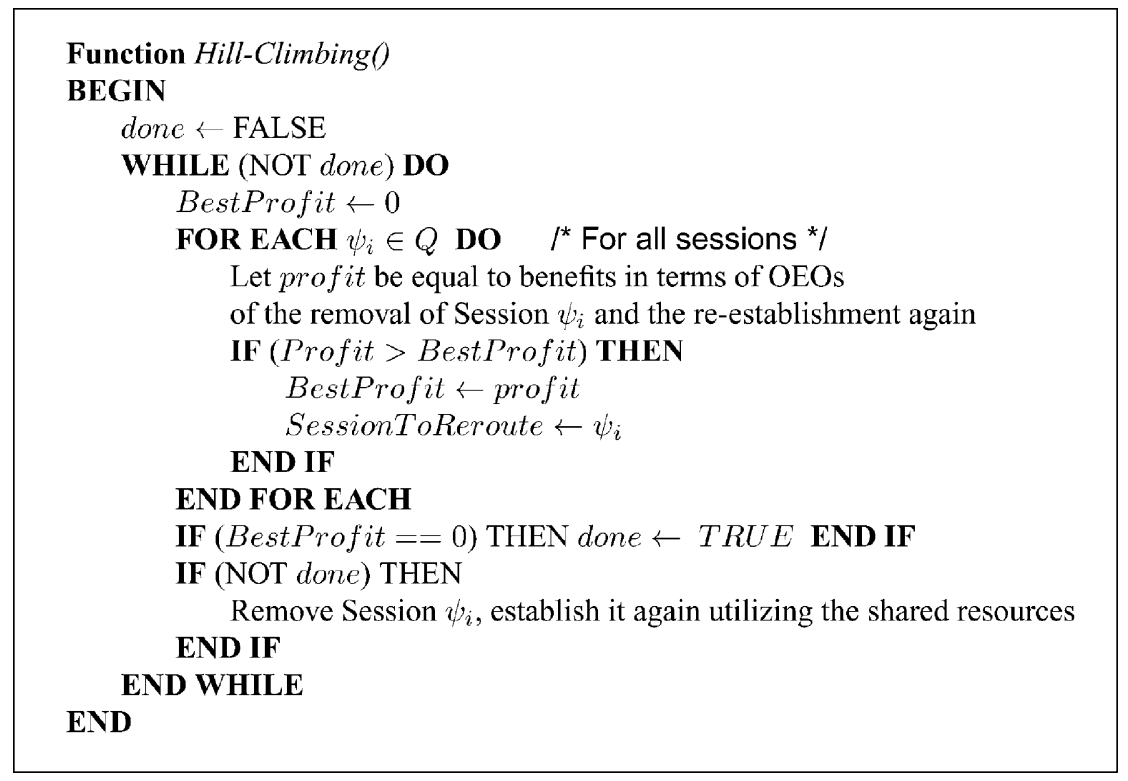

Fig. 8. Hill-climbing heuristic.

bi-directional fibers. Every switching node is assumed to have a client (e.g., an IP router) connected to it. Consider every fiber carries up to 16 channels. Also, consider that every fiber utilizes Long Haul (LH) transmission system with maximum optical reach of $400 \mathrm{~km}$. All links are assumed to be of $300 \mathrm{~km}$ length; thus requiring regeneration at every intermediate hop.

Consider a mesh virtual topology is requested to be implemented on top of this physical network. In other words, traffic demand is composed of one lightpath request from every node to every other node with 56 connection requests. We utilize three schemes for optimization of the virtual topology cost dominated by OEO devices required to clean up the signal. The first scheme is a sequential application of an optimal solution for the problem of finding a pair of fiber-disjoint paths with minimum number of total regeneration on these routes given the current configuration of the network. The optimal solution is found by using an integer-linear program (ILP) shown in the appendix. This ILP program is sequentially solved for all lightpath requests where the solution of the current request utilizes the shared resources of all previous request and is also constrained by their routing solutions. Two ILP solutions are used: ILP-Level 1 and ILP-Level 2 for the two sharing levels. The second scheme uses the H-Basic heuristic discussed in the previous section. Note that the H-Basic heuristic is similar to the 


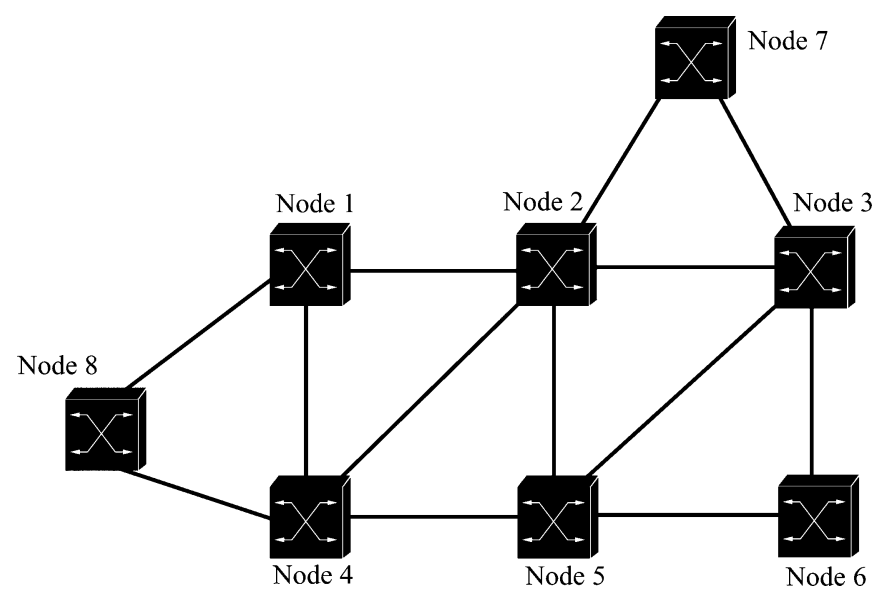

Fig. 9. A simple physical topology of an optical network. Two distinct fibers connect every two neighboring switches. A client (not shown) is attached to every switch.

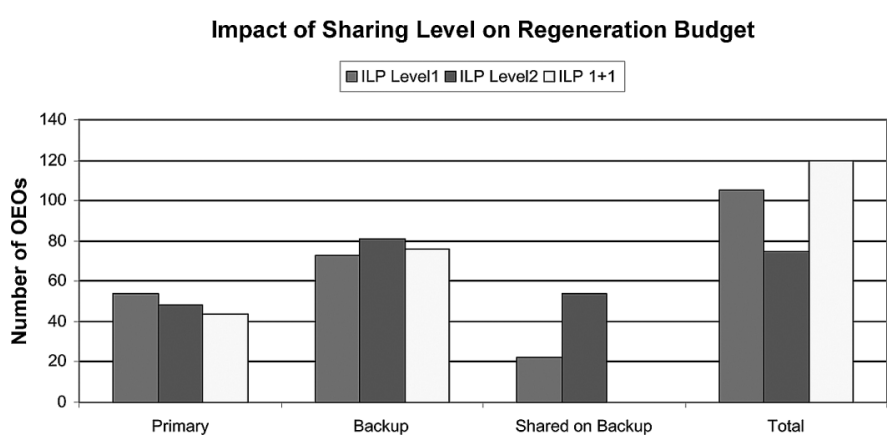

Fig. 10. Comparison between the two sharing levels and $1+1$ protection. The number of required OEOs devices is shown for working, backup, actual shared on backup, and the actual total.

first scheme, but it instead utilizes a heuristic (sub-optimal) solution for finding the pair-of disjoint paths. The third scheme uses the $\mathrm{HC}$ heuristic. Fig. 10 shows a comparison between the two sharing levels and $1+1$ protection. On the $x$-axis, we show the primary paths budget, backup budget without sharing, shared portion of backup budget, and the actual total composed of primary and backup less than the shared portion. On the y-axis, we show the number of OEOs used. We make the following observations. 1) As expected, the OEO budget for backup is larger than that for primary and it is true for all three protection schemes. This is due to the fact that the first shortest-path (primary path) forces the second shortest-path (backup path) to be longer and incurring more impairments; thus requiring more OEOs. 2) Level 2 sharing provides about $150 \%$ increase in the number of shared regenerators, compared with Level 1 sharing. 3) The total OEO budget reduction using Level 2 compared to Level 1 and $1+1$ is $29 \%$ and $38 \%$, respectively. Not only that, but even Level 1 sharing provides $13 \%$ reduction of the total OEO budget compared with $1+1$ protection scheme. These remarkable savings strongly support the concept of generalized sharing scheme. The performance in the previous figure was only taken with respect to the ILP solution. In the following, we compare the three approaches: ILP, H-Basic, and HC using Level 2. Fig. 11 shows that comparison for both the primary and the backup. As expected, the ILP provides better solution value

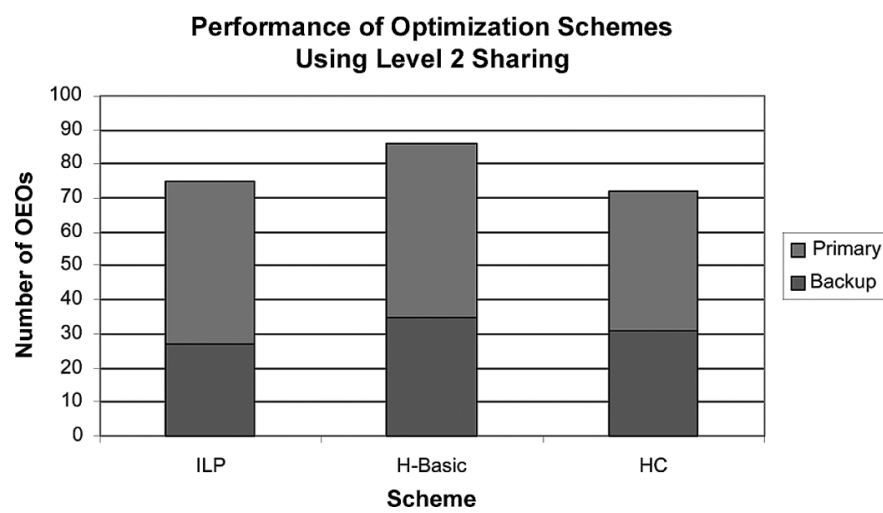

Fig. 11. Performance comparison between ILP, H-Basic, and HC with respect to the OEO budgets for primary and backup. Level 2 sharing is used.

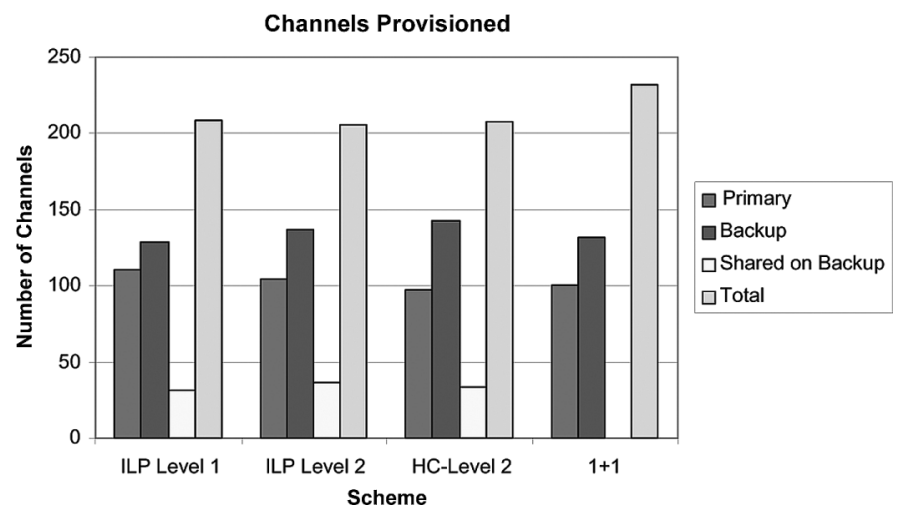

Fig. 12. Comparison between ILP-Level 1, ILP-Level 2, HC Level 2, and 1+1 protection schemes with respect to channels budget. Number of the channels is used as a comparison criterion instead of channel-km since all fibers have same length.

that the $\mathrm{H}$-Basic heuristic. However, the $\mathrm{HC}$ heuristic is able to enhance the H-Basic solution and iteratively re-routes connections in order to reduce the cost further. The cost reduction of the HC heuristic compared with the H-Basic is $16 \%$; allowing it to surpass the value of ILP. ${ }^{1}$ Not only that the HC heuristic using Level 2 is able to reduce the cost the of OEOs, but the total channels provisioned is also reduced. Fig. 12 shows the results comparing the channel budget of the four schemes: ILP-Level 1, ILP-Level 2, HC-Level 2, and 1+1. We show the budget for primary, backup, shared portion on backup, and the effective total. We notice comparable results for the four schemes. However, we observe about $11 \%$ reduction in the total channel budget using any of the shared protection schemes compared with the dedicated $1+1$ protection. Sharing requires typically $30 \%$ less channels than $1+1$, however the observed $11 \%$ reduction is most likely an artifact of the proposed architecture and algorithm because its primary objective is to reduce the number of OEOs rather than the number of channels.

\section{Results For the Italian Network}

In the previous section, we demonstrated 1) the benefits of the generalized sharing concept, 2) the superiority of Level 2 over Level 1, and 3) the performance of the HC heuristic with respect to the ILP approach for small-sized network. In this section,

\footnotetext{
${ }^{1}$ Observe that the ILP scheme is a sequential one.
} 


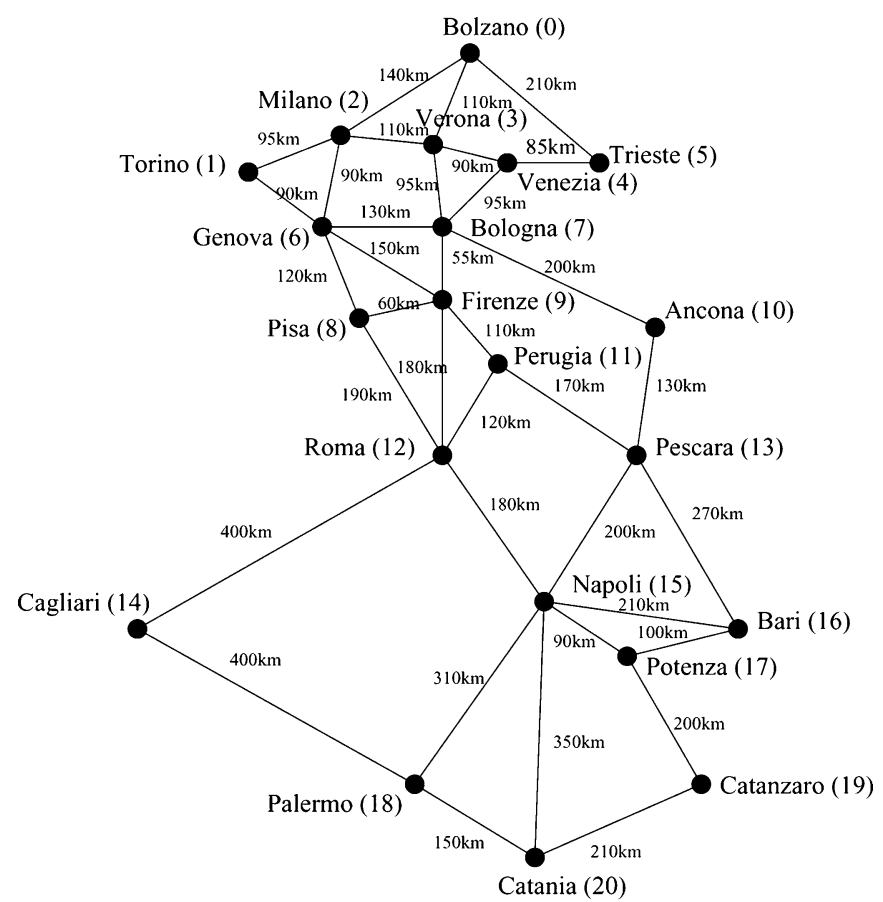

Fig. 13. Italian network topology. 21 nodes and 36 bi-directional fibers. Maximum optical reach is $400 \mathrm{~km}$. Maximum number of wavelengths on a fiber is 72 .

we investigate a larger network using only $\mathrm{HC}$ algorithm. For this reason, we consider the Italian network topology shown in Fig. 13.

We illustrate the cost of designing a full-mesh virtual topology on the Italian network. Fig. 14 shows the performance of the HC heuristic using Level 1 and Level 2 sharing. In addition, the $1+1$ solution is provided for reference. The $\mathrm{x}$-axis shows the iteration number of the heuristic and the y-axis shows the current total number of OEOs needed. At Iteration 0, we have the solution obtained by the H-Basic heuristic. At each iteration, recall that all connections are inspected to see the session providing the best benefit for re-routing. If such a profit is possible, that session is re-routed and the process is repeated. Otherwise the $\mathrm{HC}$ heuristic terminates. We observe that at the termination point of the $\mathrm{HC}$ heuristic in Iteration 75, 21\% reduction in the OEO budget is achieved using Level 2 sharing compared with the final solution of the Level 1 scheme. Also, we notice large difference of $40 \%$ reduction between Level 2 and $1+1$ dedicated protection. Notice that $1+1$ is constant since re-routing only takes advantage of newly formed sharing opportunities and no benefit in re-routing $1+1$ connections. As expected, we also notice that more reduction percentage is achieved using Level 2 than Level 1. In particular we observe $16 \%$ reduction of the H-Basic solution for Level 2 compared with only $10 \%$ reduction using Level 1 . This is expected since Level 2 has more opportunities for OEO sharing when sessions are re-routed.

Fig. 15 shows the Lambda-km budget for the two sharing levels and $1+1$ scheme. We notice that there is no significant difference between the two sharing levels (only $2 \%$ increase). The impact of this small increase in channel budget is marginalized by the significant reductions of the dominant OEO cost as

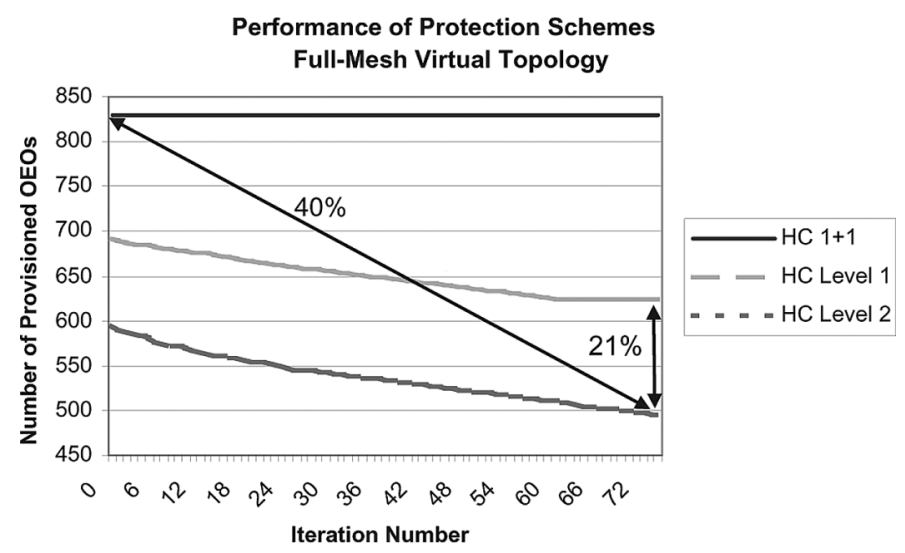

Fig. 14. Performance of the HC heuristic using Level 1, Level 2. The 1+1 solution is used for reference. The $\mathrm{x}$-axis shows the iteration number of the $\mathrm{HC}$ algorithm, while the y-axis shows the total number of provisioned OEOs. A full-mesh virtual topology is embedded with 420 connection requests.

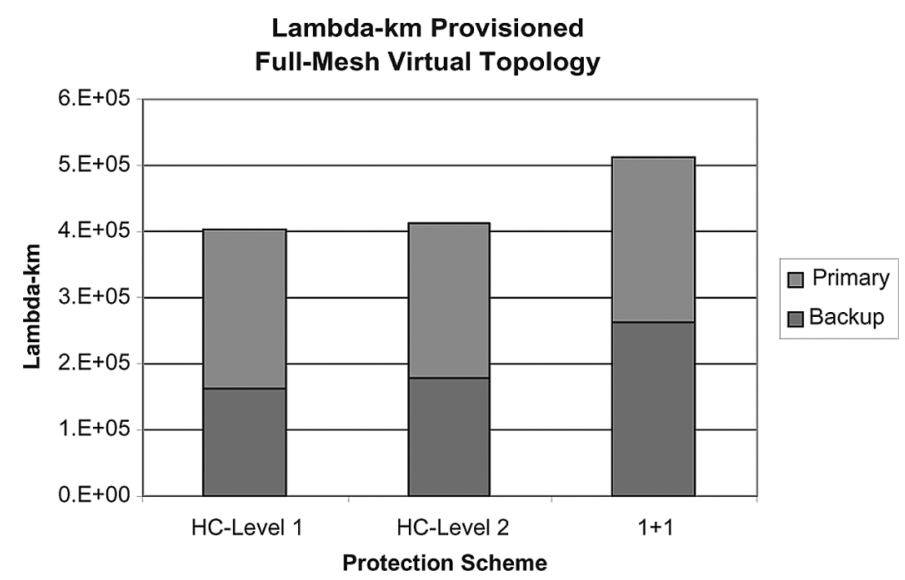

Fig. 15. Lambda-km budget for the two sharing levels and $1+1$.

demonstrated by the previous figure. We also notice about $19 \%$ reduction in Lambda-km budget compared with $1+1$ protection.

Fig. 16 shows statistics for Level 2 solution using the HC heuristic. On the $\mathrm{x}$-axis, we vary the value $X$ representing the maximum number of backup paths that can use an OEO. On the $y$-axis, we show the number of OEOs in the network that are shared by at most $X$ backup paths. The values shown are cumulative. We make the observation that the majority of backup OEOs (90\% or 134 out of 153) have at most six backup paths. Six backup paths is a relatively low number and represents about $1.5 \%$ of the total number of backup paths in the network. In general, it is beneficial not to over-share a given OEO, but rather attempt at balancing the sharing of OEOs across the network. In a dynamic environment, a given shared OEO may become unavailable for further sharing if the number of backup paths sharing that $\mathrm{OEO}$ exceeds a given threshold.

Fig. 17 shows the statistics for the sharing table size for OEOs in the network used in the same solution above. On the x-axis, we show the maximum sharing table size, $X$, in terms of number of fibers, and on the $y$-axis, we show the number of OEOs in the network whose sharing table size does not exceed $X$. Data is shown in cumulative format. We notice that about $50 \%$ of OEOs have sharing table size that does not exceed 15 fibers (or 20\% of the total fibers in the network). For the optimization of the 
Summary of the Sharing in the Network

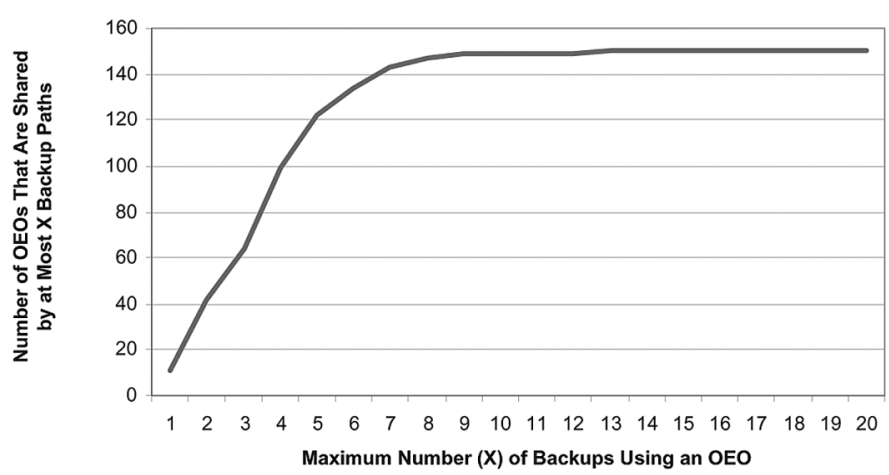

Fig. 16. Cumulative number of OEOs constrained by a maximum number for backups.

Summary of Sharing Table size

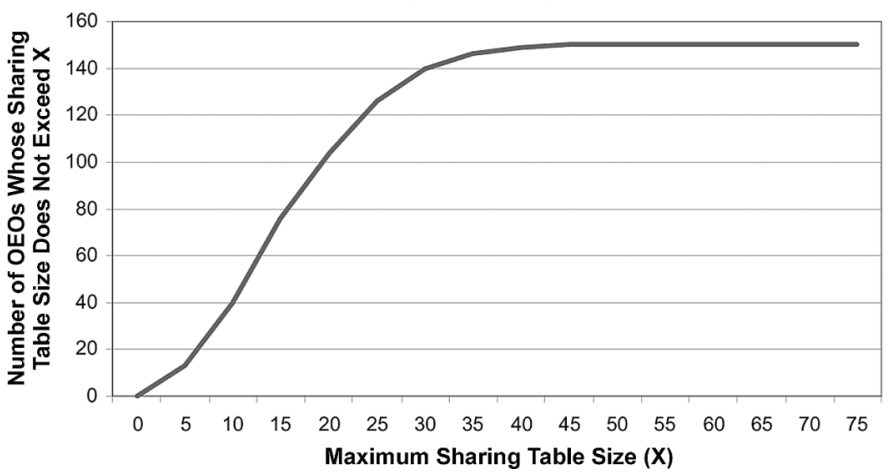

Fig. 17. Cumulative number of OEOs constrained by the maximum OEO sharing table size.

OEO sharing, the ingress node or the management tool should have access to the sharing table information. However, as the sharing table size increases, the resource management traffic increases as well, and may trigger a scalability problem. Solution for this problem include limiting the sharing table size and/or abstracting the sharing information at the OEO or the node levels. This abstraction comes at the expense of less accurate information and, hence, lower utilization of shared OEOs. The issue of balance between the amount of information related to sharing tables distributed and the utilization of network resources in dynamic environments is an important problem.

\section{Control Plane Issues}

Managing the optical and electronic resources in the network as well as the establishment and de-establishment of optical services are the main duties of the control plane. The connection request is represented by $\langle s, d, p\rangle$, where $s$ and $d$ are the ingress and egress nodes, respectively, and $p$ is the protection requirement (e.g., 0 for no protection, 1 for 1: $n$ protection, and 2 for $1+1$ protection). Given the connection request from an attached client, the intelligent optical cross-connect attempts at establishing of the optical lightpath(s). If we assume that full information about network resources is available to all cross-connects as well as no restriction on the time for the connection establishment phase, then every cross-connect can find the best route with the minimum cost. However, such assumptions are not realistic. First, the amount of bandwidth required for any link-state protocol (e.g., Open Shortest-Path First (OSPF)) for the distribution of the information pertaining to network resources is very large for real-life networks. Thus, it is expected that only few parameters related to the links between cross-connects will be distributed. Information related to wavelengths are expected to be abstracted into one or more of these link parameters (e.g., percentage of available wavelengths). Second, the computation time of the route needs to be bounded.

We consider five provisioning scenarios:

1) ILP-Full. This scheme assumes that full information about the network resources is available to every cross-connect. When there is a connection request, the cross-connect builds an integer-linear model (see the Appendix) and finds the optimal solution using an ILP solver such as CPLEX. Although this scheme produces the optimal solution, it is computationally intensive and cannot be bounded in time unless sub-optimal solutions can be used. It is used here as a means for a lower-bound.

2) Heuristic-Full This scheme also assumes full knowledge of network resource. However, it uses Dijkstra's algorithm for first finding the primary path and then the backup path as described in EstablishSession() in Section II-A.

3) ILP-Static. In this scheme, no global information is needed. Each cross-connect has a priori a set of routes to choose from. This information is available for all possible connections between any of its clients and other clients. Once a connection is requested, signaling is initiated on these routes and resource availability is collected along the paths. Once the resource availability arrives at the egress node, an ILP problem is built using this information. This problem differs from Scheme 1 above in that only links of the static route can be used in the solution, thus reducing computation time. The model is solved using CPLEX. Note that as the search space is greatly reduced from Scheme 1, usage of ILP solutions can be investigated for use in real life.

4) Heuristic-Static. This is similar to the previous scheme, however, we use the heuristic in finding the solution. First, the amount of regeneration needed on the primary path is computed. Next, the amount of regeneration on the backup route is calculated where a regenerator is shared only if no fiber in its sharing table is contained in the primary path.

5) Heuristic-Static Swap This scheme is motivated by the observation that the two routes signaled in the network were computed without knowledge of sharing resources. Thus, it might be beneficial to use the backup route as a primary and vice versa depending on the state of the sharing tables on intermediate nodes along the two routes. This schemes optimizes the previous scheme by reversing the role of the primary and backup routes. In other words, it finds two solutions. The first is the solution of Scheme 4. The second, is computed by treating primary route as a backup and the backup route as primary. Whichever requires the least number of OEOs, is used.

Before we provide evaluation of these provisioning schemes, we touch on the restoration aspect of the network. When failure 
occurs and since we consider path protection here, the network is flooded with failure alarms. In the case of a centralized network management system (NMS), the NMS signals the backup and switches the traffic to the backup path. In case of distributed provisioning, the alarm reaches the ingress node and the backup is signaled from ingress to egress. Note that contention of pre-allocated shared OEOs and wavelengths does not occur under our assumption of single-failure scenario.

\section{A. Performance of Provisioning Schemes}

We would like to compare the performance of the network under the five provisioning scenarios. In order to do that, we consider the following experiment. We consider the Italian network and the set of all possible connection pairs (420). Since we would like to observe the effect of the degree of knowledge about the OEO sharing tables on the regeneration budget, we assume that every link has one free wavelength for usage. Each connection routed is freed before the next connection pair is considered. At each node, the OEO sharing table is randomly built as follows. Given the probability of a given fiber being a member of a sharing table, we find the set of fibers belonging to a given table. This process is repeated for all 21 nodes so that all nodes have uniform OEO sharing table size, but with different fiber memberships.

Fig. 18 shows the result averaged over the traffic demand. On the $\mathrm{x}$-axis, we show the percentage of fibers in the sharing table. On the y-axis, we show the average number of OEOs per connection (both the primary and backup of that connection) for a given scenario. We make the following observations.

1) Since the ILP-Full scenario is optimal, it finds the minimum number of OEOs for a given connection pair and performs the best. The remaining scenarios come in the next place with smaller performance gaps amongst each other.

2) When the size of the sharing table is 0 (i.e., no sharing tables exist in the network), we have the highest regeneration requirement for connections with value of around two OEOs per connection. This value is almost the same for all five schemes.

3) For the case where the sharing table contains an average of seven fibers ( $10 \%$ of the 72 fibers), the OEO budget is drastically decreased to 0.67 OEOs on average for the ILP-Full solution. In second place, the Heuristic-Full scenario requires $0.74 \mathrm{OEOs}$ on average. The more realistic scenario of Heuristic-Static requires 0.98 OEOs, while the addition of the route swap feature improves the solution; thus requiring 0.92 OEOs on average.

4) When the average size of the sharing table is 15 (20\% fibers on average), we observe that the Heuristic-Static requires an increase of $60 \%$ in terms of OEOs compared with the optimal solution. With the swap feature, the increase is $47 \%$. Notice that from Fig. 17, we observed that $50 \%$ of sharing tables in the full-mesh demand do not exceed 15 fibers in size. Thus, the network designer might consider the trade-off between cost of the network and bandwidth of the link-state protocol.

5) As the OEO sharing table size increases, we observe that the performance gap of the heuristics with respect to the optimal solution widens. At the same time, it becomes

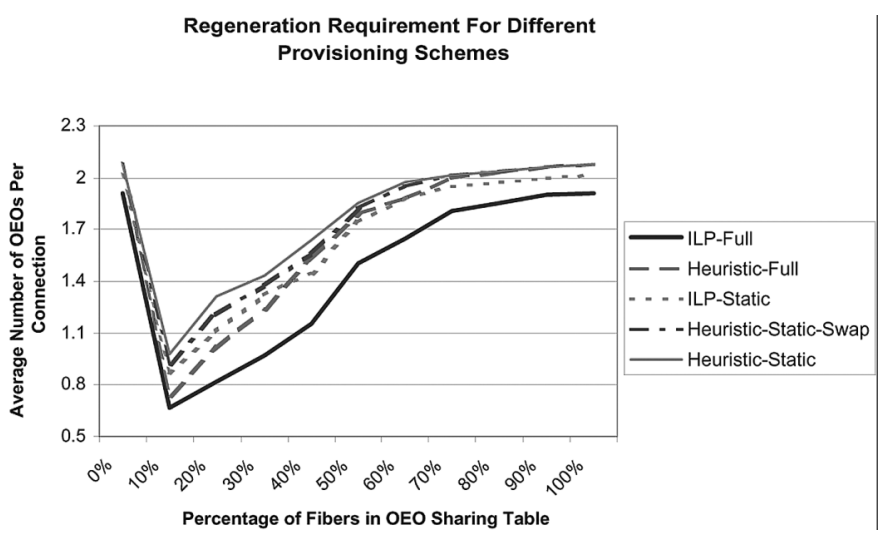

Fig. 18. Average number of OEOs per connection (primary and backup) for different provisioning schemes.

more difficult to distribute the sharing tables in the network due to bandwidth constraints on the control channel.

6) We observe when the sharing table size is increased beyond $50 \%$ of total fibers, usage of heuristic-based approaches with partial sharing information have little significance on the reduction of the network cost. However, keeping sharing tables' sizes around 20\%-30\% should allow for the enhancements of network cost.

\section{SUMMARY}

Shared Path Protection has been demonstrated to be a very efficient survivability scheme for optical networking. In this scheme, multiple backup paths can share a given optical channel if their corresponding primary routes are not expected to fail simultaneously. The focus in this area has been the optimization of the total channels (i.e., bandwidth) provisioned in the network through the intelligent routing of primary and backup routes. In this work, we extend the current path protection sharing scheme and introduce the Generalized Sharing Concept. In this concept, we allow for additional sharing of important node devices. These node devices (e.g., OEOs, pure all-optical converters, etc.) constitute the dominant cost factor in an optical backbone network and the reduction of their number is of paramount importance. For demonstration purposes, we extend the concept of $1: N$ shared path protection to allow for the sharing of electronic regenerators needed for coping with optical transmission impairments. Both design and control plane issues are discussed through numerical examples. Considerable cost reduction in electronic budget are demonstrated.

\section{APPENDIX}

In the following, we provide an ILP formulation for the PDP-OEO problem. The model is subject to the following constraints.

- Let $U P_{x, y}^{w}$ be a binary variable equal to 1 if and only if (iff) Wavelength $w$ on Fiber $(x, y)$ is used by the primary path. Similarly, $U B_{x, y}^{w}$ is defined for the backup path. The following constraints ensure that the two routes are established. For the primary path, the source has transmission

$$
\sum_{w \in W} \sum_{(s, x) \in E} U P_{s, x}^{w}=1
$$


and the destination has reception

$$
\sum_{w \in W} \sum_{(x, d) \in E} U P_{x, d}^{w}=1
$$

Similar constraints are defined for the backup path.

- At an intermediate Node $y$, the flow conservation for the primary path is observed:

$$
\sum_{(x, y) \in E} U P_{x, y}^{w}=\sum_{(y, z) \in E} U P_{y, z}^{w} \quad \forall y \in M, \forall w \in W
$$

Similarly for the backup path.

- Let $L P_{x, y}$ be a binary variable that is equal to 1 iff Fiber $(x, y)$ is used by the primary path. $L B_{x, y}$ is defined similarly for the backup path. A link is used by the primary path if one of its wavelengths is used:

$$
L P_{x, y}=\sum_{w \in W} U P_{x, y}^{w} \quad \forall x, y \in M .
$$

Similarly for the backup path.

- The primary and the backup paths must be fiber-disjoint:

$$
L P_{x, y}+L B_{x, y} \leq 1 \quad \forall x, y \in M .
$$

- The primary path can use a wavelength on a given fiber only if that wavelength is free. Let $F_{r e e}, y(w)=1$ iff Wavelength $w$ on Fiber $(x, y)$ is free. We have

$$
U P_{x, y}^{w} \leq \operatorname{Free}_{x, y}(w) \quad \forall x, y \in M, \forall w \in W .
$$

The backup path, however, can use the wavelength if it is free or used by another backup path. Let $\operatorname{Backup}_{x, y}(w)$ equal to 1 if such a condition is true, and zero otherwise. We have $\forall x, y \in$ $M, \forall w \in W$ :

$$
U B_{x, y}^{w} \leq \text { Free }_{x, y}(w) \text { OR Backup } x, y(w) .
$$

- Let $I P i n_{x, y}^{w}$ be a real-valued variable defined as the amount of impairments on Wavelength $w$ arriving at Node $x$ and destined to Fiber $(x, y)$. This variable represents the value of the accumulated impairments before any possible regeneration. Let IPout ${ }_{x, y}^{w}$ be a real-valued variable defined as the amount of impairments out of Node $x$ (after a possible regeneration) on Fiber $(x, y)$ and Wavelength $w . I B i n_{x, y}^{w}$ and IBout ${ }_{x, y}^{w}$ are defined similarly for the backup path. The signal at the output of the Source $s$ is fresh and has zero impairments for the primary:

$$
\operatorname{IPin}_{s, y}^{w}=0, \quad \text { IPout }_{s, y}^{w}=0 \quad \forall(s, y) \in E, \forall w \in W .
$$

and similarly for the backup path.

- Let $I P i n_{x}^{w}$ be a real-valued variable defined as the amount of impairments on Wavelength $w$ input to Node $x$ on the primary path. $I B i n_{x}^{w}$ is defined similarly for the backup path. Let the contribution of impairments of Fiber $(x, y)$ be given by $I(x, y)$.
The amount of impairments at a given node for the primary path is given by the following constraint. We have $\forall x \in M, \forall w \in W$

$$
\operatorname{IPin}_{x}^{w}=\sum_{(y, x) \in E}\left(\operatorname{IPout}_{y, x}^{w}+I(y, x) \times U P_{y, x}^{w}\right) .
$$

$\operatorname{IBin}_{x}^{w}$ is given similarly.

- A given wavelength on a given fiber has zero impairments unless it is used. For the primary path, we have

$$
I P i n_{x, y}^{w} \leq U P_{x, y}^{w} \quad \forall x, y \in M, \forall w \in W .
$$

Similar constraint is defined for the backup path.

- The amount of impairments input to Node $x$ on Wavelength $w$ for the primary path is transferred to one of the output fibers as follows:

$$
\sum_{(x, y) \in E} \operatorname{IPin}_{x, y}^{w}=I P i n_{x}^{w} \quad \forall x \in M, \forall w \in W .
$$

Similarly for the backup path.

- The amount of impairments out of Node $x$ on Wavelength $w$ to Fiber $(x, y)$ for the primary path is given by

$$
\operatorname{IPout}_{x, y}^{w}=I P i n_{x, y}^{w}-\epsilon P 1_{x, y}^{w} \quad \forall x, y \in M, \forall w \in W
$$

where $0 \leq \epsilon P 1_{x, y}^{w} \leq 1$. A non-zero value of $\epsilon P 1_{x, y}^{w}$ indicates that a regenerator is installed to clean up the signal.

- Let $R P_{x, y}^{w}$ be a binary variable. $R P_{x, y}^{w}=1$ indicates that a regenerator is used at Node $x$ on Wavelength $w$ to clean up the primary signal routed on Fiber $(x, y)$. Also, let $\epsilon P 2_{x, y}^{w}, 0 \leq$ $\epsilon P 2_{x, y}^{w}<1$ be a real-valued variable. $R P_{x, y}^{w}$ can be given by

$$
R P_{x, y}^{w}=\epsilon P 2_{x, y}^{w}+\epsilon P 1_{x, y}^{w} \quad \forall x, y \in M, \forall w \in W .
$$

If $\epsilon P 1_{x, y}^{w}$ is chosen to be greater than 0 , in order to reduce the output impairment (see Constraint 12), then $\epsilon P 2_{x, y}^{w}$ variable must be equal to $\left(1-\epsilon P 1_{x, y}^{w}\right)$. This is due to the fact that $R P_{x, y}^{w}$ is binary variable and can hold only two distinct values: 0 or 1 . On the other hand, if $\epsilon P 1_{x, y}^{w}$ is set to 0 , indicating no regeneration, then $\epsilon P 2_{x, y}^{w}$ variable must be equal to 0 in order for $R P_{x, y}^{w}$ to be equal to 0 . Similar logic applies to the backup path.

- A regeneration at Node $x$ and Wavelength $w$ for the primary path is given by the contribution of all output fibers to the Node $x$ and is given by

$$
R P_{x}^{w}=\sum_{(y, x) \in E} R P_{y, x}^{w} \quad \forall x \in M, \forall w \in W .
$$

$R B_{x}^{w}$ is defined similarly for the backup path.

- For Level 2 sharing, let $P_{x}^{w}$ be the set of all fiber links prohibiting the sharing of the regenerator on Wavelength $w$ at Node $x$. In other words, it is the union of the primary routes for all backup paths sharing this regenerator. Let $C_{x}^{w}$ be a binary variable equal to 1 iff the regenerator cannot be shared. Also, 
consider the real-valued variable $\epsilon_{x}^{w}, 0 \leq \epsilon_{x}^{w}<1$. $C_{x}^{w}$ can be written as

$$
C_{x}^{w}=\frac{1}{\left|P_{x}^{w}\right|}\left(\sum_{(a, b) \in P_{x}^{w}} L P_{a, b}\right)+\epsilon_{x}^{w} \quad \forall x \in M, \forall w \in W .
$$

If $P_{x}^{w}$ is empty, $C_{x}^{w}$ is set to 1 . In the case that $\left|P_{x}^{w}\right|>0$, we note that the right-hand expression in the above formula (excluding $\epsilon_{x}^{w}$ ) can range from 0 (i.e., primary does not visit any link in $P_{x}^{w}$ ) and 1 (i.e., primary visits every link in $P_{x}^{w}$ ). If the value is 0 , the only valid value for $\epsilon_{x}^{w}$ is 0 . This is due to the fact that $C_{x}^{w}$ is a binary variable and $\epsilon_{x}^{w}$ cannot have a value greater or equal to 1 . If the value of the expression is 1 , then $\epsilon_{x}^{w}$ must be 0 and $C_{x}^{w}$ is set to 1 . Otherwise, if the value of the expression is $t, 0<t<1$ (i.e., primary visits $t \times\left|P_{x}^{w}\right|$ links of $P_{x}^{w}$ ), then $\epsilon_{x}^{w}$ has only one valid value $(1-t)$ that sets $C_{x}^{w}$ to be 1 . In other words, $\epsilon_{x}^{w}$ completes $t$ to the value 1 . In all three cases, $C_{x}^{w}$ is set to a value that is consistent with its definition.

- Let $H_{x}^{w}$ be a binary variable, where $H_{x}^{w}=1$ iff there is a new (non-shared) regenerator used at Node $x$ and Wavelength $w$ for the backup path. Let $\epsilon 3_{x}^{w}$ be a real-valued variable, $-0.5 \leq \epsilon 3_{x}^{w} \leq 0$. Clearly, $H_{x}^{w}$ should be equal to 1 only if there is a need for a regenerator and that regenerator cannot be shared (i.e., the primary path visits at least one of the fibers in $P_{x}^{w}$; hence a regenerator needs to be installed). The following equation captures this logic:

$$
H_{x}^{w}=0.5 R B_{x}^{w}+0.5 C_{x}^{w}+\epsilon 3_{x}^{w} \quad \forall x \in M, \forall w \in W .
$$

There are four cases:

1) The backup path does not need a regenerator at Node $x$ using Wavelength $w$ and the regenerator at Node $x$ and Wavelength $w$ cannot be shared. In this case $R B_{x}^{w}=0$ and $C_{x}^{w}=1$. In this case, the only valid value for $\epsilon 3_{x}^{w}$ is set to -0.5 and $H_{x}^{w}$ is set to 0 .

2) If a regenerator is needed and that regenerator cannot be shared, both $R B_{x}^{w}$ and $C_{x}^{w}$ are set to 1 ; thus the only valid value for $\epsilon 3_{x}^{w}$ is 0 and $H_{x}^{w}$ is set to 1 .

3) If a regenerator is not needed and that regenerator can be shared, both $R B_{x}^{w}$ and $C_{x}^{w}$ are 0 and $\epsilon 3_{x}^{w}$ must be equal to 0 ; thus $H_{x}^{w}$ is set to 0 .

4) If a regenerator is needed and that regenerator can be shared, then $R B_{x}^{w}=1$ and $C_{x}^{w}=0$; thus $\epsilon 3_{x}^{w}$ is -0.5 and $H_{x}^{w}$ is set to 0 .

In all four possible cases $H_{x}^{w}$ is set to a value consistent with its definition.

- If Level 1 sharing is used, we have the following. Let $O_{x}^{w}$ be a binary variable equal to 1 iff the input backup signal uses Wavelength $w$ and is OEO terminated at Node $x$ (by another backup signal). Let $I S T \operatorname{Trm}_{x, y}(w)$ be equal to 1 if the backup Wavelength $w$ on Fiber $(x, y)$ is terminated by an OEO at Node $y . O_{x}^{w}$ is given by

$O_{x}^{w}=\sum_{(y, x) \in E}\left(\operatorname{ISTerm}_{y, x}(w) \times U B_{y, x}^{w}\right) \forall x \in M, \forall w \in W$.

Since we are guaranteed that a node receives at most one input signal for a given connection, $O_{x}^{w}$ can be set by the above expression to either 0 or 1 . If it is set to 0 , that means either input wavelength is not OEO terminated or this node is not part of the solution. On the other hand, if the value of $O_{x}^{w}$ is equal to 1 , then the input signal is OEO terminated. In both cases $O_{x}^{w}$ is set to a value consistent with its definition. $H_{x}^{w}$ can now be written as

$H_{x}^{w}=0.5 R B_{x}^{w}+0.5 \times\left(1-O_{x}^{w}\right)+\epsilon 3_{x}^{w} \quad \forall x \in M, \forall w \in W$.

Going through all the four different combination of value of $R B_{x}^{w}$ and $O_{x}^{w}$ as was shown for Level 2 logic, one can see that in all four possible cases of Level 1 logic, $H_{x}^{w}$ is set to a value consistent with its definition.

- Let $S_{x, y}^{w}$ be a binary variable equal to 1 iff Wavelength $w$ on Fiber $(x, y)$ cannot be shared with the backup path. A wavelength can be in one of three different states: 1) free; 2) used by a primary path; and 3) used by a backup path. For the first two cases, $S_{x, y}^{w}$ is set to 1 . Let the set $F_{x, y}^{w}$ be the union of the links of all primary routes whose backups share Wavelength $w$ on Fiber $(x, y)$. Also, let $\epsilon 4_{x, y}^{w}$ be a real-valued variable, $0 \leq \epsilon 4_{x, y}^{w}<1$. $S_{x, y}^{w}$ is given by

$S_{x, y}^{w}=\frac{1}{\left|F_{x, y}^{w}\right|}\left(\sum_{(a, b) \in F_{x, y}^{w}} L P_{a, b}\right)-\epsilon 4_{x, y}^{w} \forall x, y \in M, \forall w \in W$.

The above formula follows similar logic to Constraint 15 .

- Let $K_{x, y}^{w}$ be a binary variable equal to 1 iff a new Wavelength $w$ is reserved on Fiber $(x, y)$ by the backup path. Let $\epsilon 5_{x, y}^{w}$ be a real-valued variable, $-0.5 \leq \epsilon 5_{x, y}^{w} \leq 0 . K_{x, y}^{w}$ can be written as follows:

$$
K_{x, y}^{w}=0.5 U B_{x, y}^{w}+0.5 S_{x, y}^{w}+\epsilon 5_{x, y}^{w}
$$

$K_{x, y}^{w}$ will be set to 1 only if it is used and cannot be shared (i.e., must be provisioned).

- The objective is to minimize the total number of regenerators needed in the routing of the session for both primary and backup routes:

$$
\text { Minimize } \sum_{x \in M} \sum_{w \in W}\left(R P_{x}^{w}+H_{x}^{w}\right) .
$$

\section{REFERENCES}

[1] R. Ramaswami and K. N. Sivaraja, Optical Networks: A Practical Perspective, 2nd ed. San Francisco, CA: Morgan Kaufmann, 2002.

[2] S. Ramamurthy and B. Mukherjee, "Survivable WDM mesh networks. Part I-Protection," in Proc. IEEE INFOCOM 1999, pp. 744-751.

[3] D. Elie-Dit-Cosaque, M. Ali, and L. Tancevski, "Informed dynamic shared path protection," in Proc. Optical Fiber Communication Conf. and Exhibit, Mar. 2002, pp. 492-493.

[4] S. Gangxiang, S. Bose, C. Hiang, and L. Chao, "Designing WDM optical network for reliability: routing light paths efficiently for path protection," in Proc. Optical Fiber Communication Conf., 2000, pp. 50-52.

[5] C. Ou, J. Zhang, H. Zang, L. Sahasrabuddhe, and B. Mukherjee, "New and improved approaches for shared-path protection in WDM mesh networks," J. Lightw. Technol., vol. 22, no. 5, pp. 1223-1232, May 2004.

[6] C. Ou, H. Zang, N. Singhal, K. Zhu, L. Sahasrabuddh, R. MacDonald, and B. Mukherjee, "Subpath protection for scalability and fast recovery in optical WDM mesh networks," IEEE J. Sel. Areas Commun., vol. 22, no. 9, pp. 1859-1875, Nov. 2004.

[7] P. Ho, "Segment shared protection in WDM networks with partial wavelength conversion," IEEE Commun. Lett., vol. 8, no. 6, pp. 394-396, Jun. 2004. 
[8] D. Xu, Y. Xiong, and C. Qiao, "Novel algorithms for shared segment protection," IEEE J. Sel. Areas Commun., vol. 21, no. 8, pp. 1320-1331, Oct. 2003.

[9] C. Ou, K. Zhu, H. Zang, L. Sahasrabuddhe, and B. Mukherjee, "Traffic grooming for survivable WDM networks-shared protection," IEEE $J$. Sel. Areas Commun., vol. 21, no. 9, pp. 1367-1383, Nov. 2003.

[10] L. Berthelon et al., "Implementation of all-optical networks," in Proc. 28th Eur. Conf. Optical Communication (ECOC 2002), Sep. 2002, pp. $1-2$, invited paper.

[11] I. Chlamtac, A. Ganz, and G. Karmi, "Lightpath communications: an approach to high-bandwidth optical WAN's," IEEE Trans. Commun., vol. 40, no. 7, pp. 1171-1182, Jul. 1992.

[12] J. Strand, A. L. Chiu, and R. Tkach, "Issues for routing in the optical layer," IEEE Commun. Mag., vol. 39, no. 2, pp. 81-87, Feb. 2001.

[13] M. Ali, Transmission-Efficient Design and Management of Wavelength-Routed Optical Networks. Norwell, MA: Kluwer Academic Publishers, 2001.
[14] R. Bhandari, Survivable Networks: Algorithms for Diverse Routing. Norwell, MA: Kluwer Academic, 1999.

[15] D. Xu, Y. Xiong, C. Qiao, and G. Li, "Trap avoidance and protection schemes in networks with shared risk link groups," J. Lightw. Technol., vol. 21, no. 11, pp. 2683-2693, Nov. 2003.

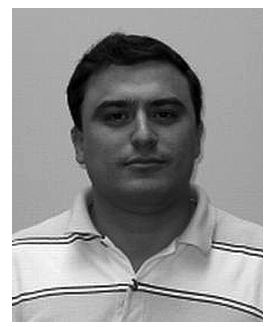

Maher Ali (M'98-SM'06) received the M.B.A. degree from the University of Texas at Dallas and the $\mathrm{Ph} . \mathrm{D}$. degree in computer science from the University of Nebraska-Lincoln.

$\mathrm{He}$ is currently with Alcatel's CTO in Plano, Texas. His work is focused on network and traffic engineering in metro Ethernet and Optical networks.

Dr. Ali is a member of the IEEE Communications Society. 